\title{
Effect of Mechanical Loading and Increased Gap on the Dynamic Response of Multiple Degree of Freedom Electrostatic Actuator
}

\author{
Hussam Kloub \\ Mechanical and Me dical Enginee ring De partment, Furtwangen University, Robert-Gerwig-Platz 1, 78120 \\ Furtwangen, Germany; hussam.kloub@hs-furtwangen.de; Tel.: +49-7723-920-2944
}

\begin{abstract}
A novel monolithic structural design of electrostatic actuator with multiple degree of freedom is presented as an approach for a system that is capable of performing scalable stroke and large electrostatic force beyond $\mathrm{mN}$ range. An electromechanical system model based on Simulink softwarew as developed for a proposed design of the electrostatic actuator. The dynamic response of the actuator was simulated and the mechanical bouncing response due to effect of realizing extra mechanical stoppers or passivation layer was investigated. Also, the mechanical bouncing as well as steady state response of the actuator w as investigated under various mechanical loading values. The results showed that the switching time increased as the mechanical load was increased. Also, bouncing maximum peak increased as the collision force w as increased.
\end{abstract}

Keywords: Inchworm motor; electrostatic micromotor; Miniaturized Motor; Cooperative electrostatic microactuators

\section{Introduction}

Electrostatic inchworm motor based on gap-closing variable capacitor provides potential solution for larger force actuation compared to area overlapping one. Unlike the constant electrostatic force in area overlapping variable capacitor, the generated electrostatic force in gap-closing variable capacitor increases as the displacement is increased. However, due to the pull-in phenomena the system stability and controllability is critical design challenges.

Various designs of complex electrostatic actuators based on gap-closing variable capacitor were developed as linear inchworm motor [1-4]. However, the force actuation capability is still in $\mathrm{mN}$ range. In [1] the electrostatic inchw orm consists of four microactuators, tw o for holding function and two for twisting function. The inchw orm is capable to perform a $100 \mathrm{~nm}$ step displacement against $0.12 \mathrm{mN}$ load, when the corresponding voltage gate is polarized at $15 \mathrm{~V}$. In [2] a displacement amplification mechanism was employed in the electrostatic inchworm design. A stroke of $35 \mu \mathrm{m}$ stroke against $0.11 \mathrm{mN}$ load w as achieved, providing that actuator w as electrically polarized at $16 \mathrm{~V}$. In [3] a shuttle with flexible arm design was implemented as electrostatic inchworm. The device performed a stroke of $10 \mu \mathrm{m}$ against $1.88 \mathrm{mN}$, when it w as electrically polarized at $110 \mathrm{~V}$. Higher load forces up to $3.7 \mathrm{mN}$ could be measured, how ever snapping failure was observed. In [4] an optimized shuttle design compared to the one in [3] was realized. The new device could perform a $1 \mu \mathrm{m}$ stroke against $1.3 \mathrm{mN}$, when it was electrically polarized at $60 \mathrm{~V}$.

In this paper, a novel monolithic structural design of electrostatic actuator with multiple degree of freedom is presented as an approach for a system that is capable of performing scalable stroke and large electrostatic force beyond $\mathrm{mN}$ range. The actuator is a kind of mechanical oscillator which can be driven in the xy-directions by three voltage electrodes. Multiple actuators can be used to increase the overall applied electrostatic force.

\section{Methods}




\subsection{Structural Design and Operation Concept}

The top view of the electrostatic actuator is shown in Figure 1. The actuator consists mainly of mechanical oscillator and voltage electrodes. The mechanical oscillator is the square shape frame, suspended by mechanical springs with anchor at the center. The frame is designed to be movable in the in-plane directions. Mechanical stoppers are used to limit the deflection distance in the in-plane directions. The limit is designed such that the frame does not collide with the fixed electrodes, which is called here actuation gate. The mechanical oscillator with the actuation gates represent three variable capacitors, where the frame represents the movable electrode, while the actuation gate is the fixed electrode. The mechanical oscillator is electrically actuated by the lateral and vertical actuation gates. The frame itself is connected to ground through the anchor, while the actuation gate is connected to a specific voltage value. For example, when the potential voltage is applied at the vertical actuaion gate, while other actuation gates as well as the anchor are connected to ground, then electrostatic force will be generated causing the frame to be deflected tow ard the vertical actuation electrode. A comb structure called integrated shaft is a movable mechanical element used for actuating the external mechanical load. The interdigitated shaft is initially integrated with another fixed comb structure called stationary holder. The interdigitated shaft is also actuated based on electrostatic force by implementing extra voltage electrode, such that the shaft can be released from the stationary holder, but simultaneously overlapped with the comb structure of the lower part of the frame. In this case, the shaft can be deflected laterally in both directions, and therefore actuating the external mechanical load.

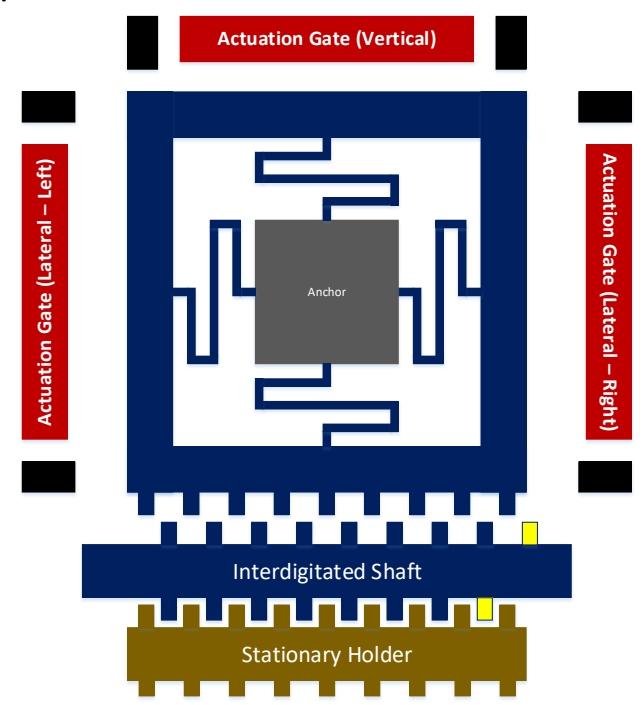

Figure 1. Top view of multiple degree of freedom electrostatic actuator design.

\subsection{Mathematical Modelling and Simulink Model}

The mathematical equations of the electrostatic device in Figure $\mathbf{1}$ are extracted based on Newton's second law. These equations will be used then to implement a Simulink model for the simulation. Here, only the mechanical oscillator, mechanical stoppers and actuation electrodes are considered for the modelling. The mechanical oscillator consists of mass $(\mathrm{m})$ realized by the frame and folded beams suspension, elastic elements of stiffness constant (k) realized by the folded beams suspension. The mechanical stoppers can be represented as elastic elements with extremely large stiffness constant $\left(\mathrm{k}_{\mathrm{s}}\right)$. The effect of mechanical stopper will be take place once the frame approaches the mechanical stopper. The dielectric medium between the frame and actuation gates affects the response of the mechanical oscillator through the viscousity damping effect. Here, vacuum with arbitrary damping coefficient (b) is applied. Only the lateral deflection response will be studied. Therefore, the internal net force (Fint) of the mechanical oscillator can be represented in terms of mass acceleration (a), velocity (v), deflection ( $x$ ) by the following equation:

$$
F_{\text {int }}=m a+b v+k x+k_{s} x_{s 1}+k_{s} x_{s 2},
$$


the parameters $x_{s 1}$ and $x_{s 2}$ are deflection limits in left and right sides of the frame. The mechanical oscillator is electrically excited by the actuation electrodes, at which electrostatic forces are generated. The lateral deflection of the frameimplies a gap closing capacitance variation. Therefore, the external excitation force $\left(F_{e x t}\right)$ can be given by the following equation:

$$
F_{\text {ext }}=0.5\left(\frac{V_{C 2}^{2} C_{2}}{G_{C 2}-x}-\frac{V_{C 1}^{2} C_{1}}{G_{C 1}+x}\right)-F_{L}
$$

the equation above represents the net electrostatic force of the right and left actuation electrodes subtracted from the external mechanical load $\left(\mathrm{F}_{\mathrm{L}}\right)$. The equation is extracted in terms of applied voltage $(\mathrm{V})$, deflection $(\mathrm{x})$, initial gap $(\mathrm{G})$ and instantaneous capacitance $(\mathrm{C})$, which are given by:

$$
C_{1}=\frac{\varepsilon_{o} \varepsilon_{r} L H}{G_{C 1}+x}, C_{2}=\frac{\varepsilon_{o} \varepsilon_{r} L H}{G_{C 2}-x},
$$

the capacitance equations are represented in terms of vacuum electric permittivity $\left(\varepsilon_{0}\right)$, dielectric constant $\left(\varepsilon_{\mathrm{r}}\right)$, electrode length $(\mathrm{L})$, electrode height $(\mathrm{H})$.

The mathematical model was implemented as Simulink block diagram in Figure 2. The model w as simulated with $\mathrm{m}=4 \mu \mathrm{g}, \mathrm{k}=34 \mathrm{kN} / \mathrm{m}, \mathrm{ks}=34 \times 10^{12} \mathrm{~N} / \mathrm{m}, \mathrm{b}=0.1 \mathrm{~N} . \mathrm{s} / \mathrm{m}, \mathrm{L}=250 \mu \mathrm{m}, \mathrm{H}=50 \mu \mathrm{m}$, $\mathrm{Gc}_{\mathrm{c}}=4.5 \mu \mathrm{m}$ and $\mathrm{Gs}=2.5 \mu \mathrm{m}$. For these values the pull-in voltage is $2.88 \mathrm{kV}$. The model contains a specific function blocks for determining the instant of mechanical impact with the mechanical stopper and the external mechanical force. In the simulation only one actuation gate $V_{C 2} w_{\text {as activated at } 10}$ KV step signal.

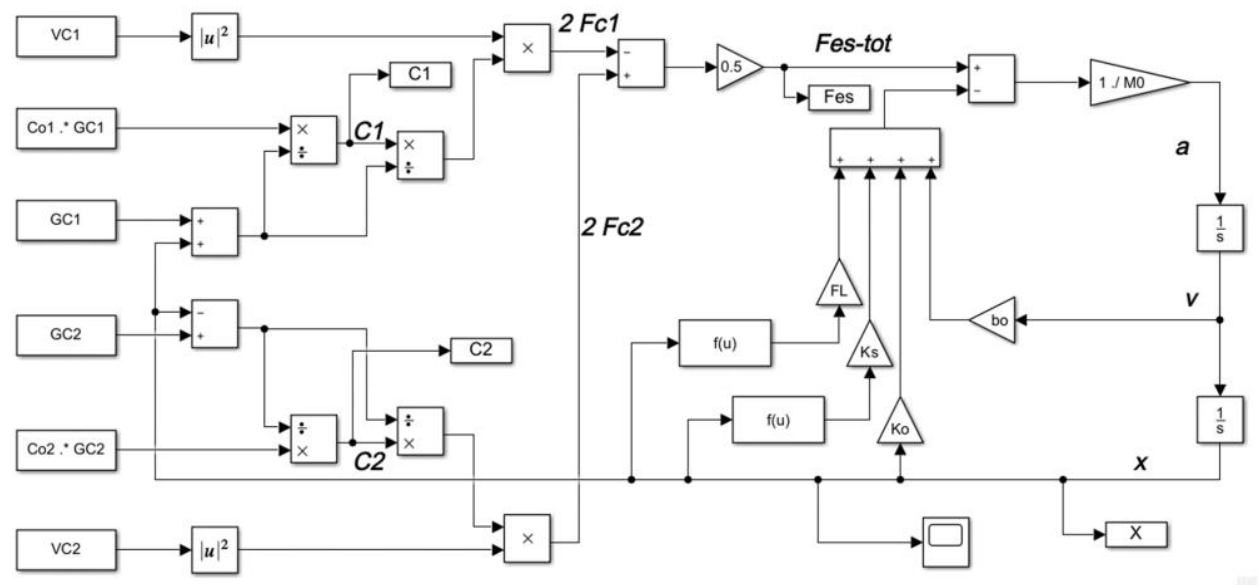

Figure 2. Simulink model of the multiple degree of freedome lectrostatic actuator.

\section{Results and Discussion}

\subsection{Effect of Mechanical Stoppers and External Mechanical Loading}

In this simulation the deflection limit due to mechanical stoppers was designed at $2.5 \mu \mathrm{m}$. The external mechanical force was initially set $2 \mu \mathrm{m}$ apart from the actuator. The dynamic response of the actuator was then extracted for various external mechanical loading, from noload, up to $2.4 \mathrm{~N}$ (Figure 3). Mechanical bouncing and mechanical damping are the main observations in this results. Mechanical bouncing maximum peak decreases as the counter mechanical loading value increases. For example, at no load the bouncing maximum peak w as $112.5 \mathrm{~nm}$, while it was $25 \mathrm{~nm}$ at $0.9 \mathrm{~N}$ load. Such bouncing response will cause actuation stability problem if the electrodes of the comb structures are included in the model. The bouncing maximum peaks will influence the design of the gap between electrodes of the comb structures. 


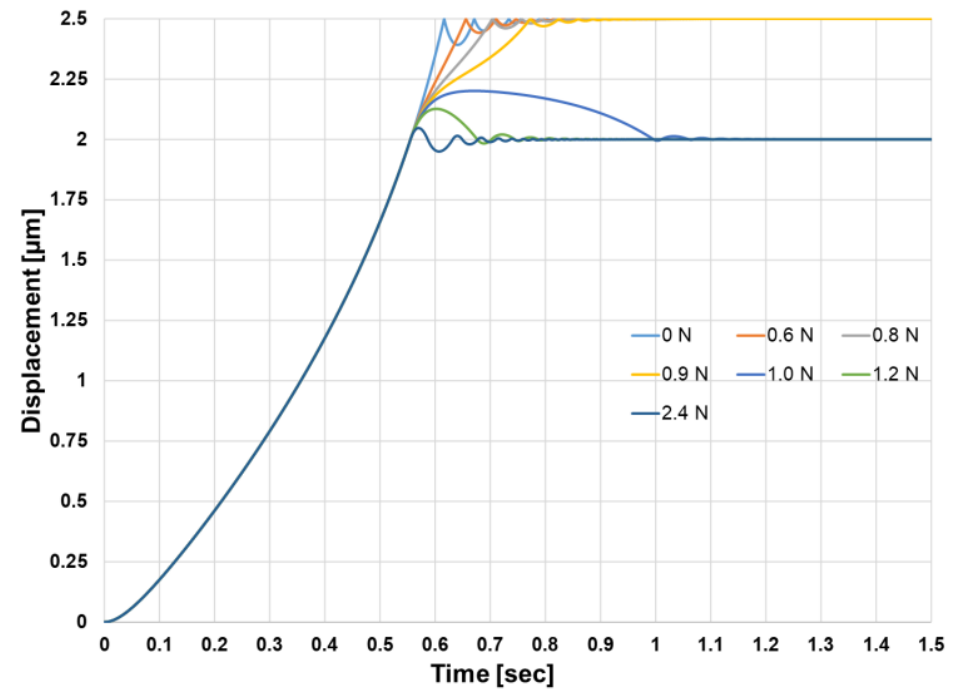

Figure 3. Dyna mic response of electrostatic actuator under diffe rent mechanical loa ding values.

Bouncing responses exhibited also different slopes, such that the larger the mechanical load, the less slope of displacement response. This decreased slope means that the mass velocity as well as kinetic energy is decreasing with increased mechanical load, thus a less collision effect appeared as a lower bouncing peak. The application of counter electrostatic force through the other variable capacitor $\left(\mathrm{C}_{1}\right)$ will be a potential approach to reduce the bouncing effect, and therefore enhancing the actuation stability of the actuator.

The maximum displacement of $2.5 \mu \mathrm{m}$ w as achieved between 0 and $0.9 \mathrm{~N}$ mechanical load. At 1 $\mathrm{N}$ load the actuator performed for a specific time further displacement before it started returning back to the $2 \mu \mathrm{m}$ position at which the mechanical load w as initially set. At the instant of load impact, the net excitation force; electrostatic and load forces decreases abruptly, thus both acceleration and velocity decreases too. When the velocity becomes zero, the mechanical load force dominates the excitation force such that the displacement starts decreasing. At this moment, the resultant electrostatic force decreases too. The final state is at which the actuator bounces with extremely small peaks around the $2 \mu \mathrm{m}$ position.

In terms of bouncing transient time, a value of $900 \mathrm{msec} w$ as roughly the same for mechanical load between 0 and $0.9 \mathrm{~N}$. For a larger loading than the $0.9 \mathrm{~N}$, the bouncing transient time was between 0.8 and $1.2 \mathrm{sec}$. The bouncing transient time will influence the design of switching signal of the actuator.

\subsection{Dynamic Response Under Increased Gap Design}

In this simulation, the mechanical stoppers were removed from the design. An electrical insulation layer passivation of $100 \mathrm{~nm}$ on the fixed electrode of the variable capacitor $\mathrm{w}$ as considered. This design allows larger travelling response (Figure 4). The response was obtained under no mechanical loading. The mechanical bouncing maximum peak increased from $112.5 \mathrm{~nm}$ to $400 \mathrm{~nm}$ compared to the case of applying extra mechanical stoppers. The larger electrostatic force is thelarger corresponding actuation force. How ever, more impact of mechanical bouncing was observed. This trade-off result will affect the design of the comb structures for the clutching. 


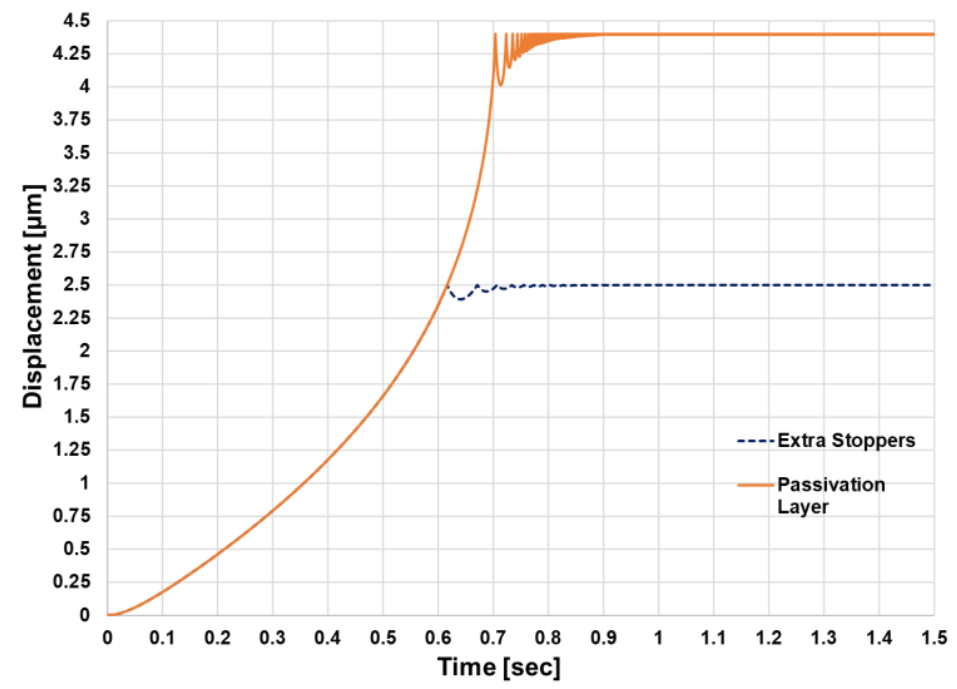

Figure 4. Dynamic re sponse of electrostatic actuator under different limiting positions.

\section{Conclusions}

In this work the effect of mechanical bouncing and mechanical damping due to mechanical collisions of the electrostatic actuator was studied by means of electromechanical Simulink model. The variation of applied mechanical load affects the resultant bouncing maximum peak, such that the larger the mechanical load the less the bouncing maximum peak. On the other hand, the displacement slope response decreases as the applied mechanical load is increase. These findings affect critically the design of electrodes of comb structures for clutching and the design of switching signal. The application of complemantary variable capacitor will be a potential approach to reduce the bouncing effect, and therefore enhancing the actuation stability of the actuator.

Acknowledgments: This project is funded by the German Research Foundation (DFG) under the umbrella of the priority programm SPP 2206 - KOMMMA (Cooperative Multistage Multistable Micro Actua tor Systems).

Conflicts of Interest: The founding sponsors had no role in the design of the study; in the collection, analyses, or interpretation of data; in the writing of the manuscript, and in the decision to publish the results.

\section{References}

1. Kim, S.-H.; Hwang, I.-H.; Jo, K.-W.; Yoon, E.-S.; Lee, J.-H. High re solution inchworm linear motor based on electrostatic twisting microactuators. J. Micromech. Microeng. 2005, 15, 1674-1682, DOI 10.1088/09601317/15/9/009.

2. Erismis, M. A.; Neves, H. P.; Puers, R.; Hoof, C. V. A low voltage large displacement large force inchworm actuator. JMEMS 2008, 17, 6, 1294-1301, DOI 10.1109/JMEMS.2008.2004852.

3. Penskiy, I.; Bergbreiter, S. Optimized electrostatic inchworm motors using a flexible driving arm. J. Micromech. Microeng. 2013, 23, 015018, DOI 10.1088/0960-1317/23/1/015018.

4. Saito, K.; Contreras, D. S.; Takeshiro, Y.; Okamoto, Y.; Hirao, S.; Nakata, Y.; Tanaka, T.; Kawamura, S.; Kaneko, M.; Uchikoba, F.; Mita, Y.; Pister, K. S. J. Study on electrostatic inchworm motor device for a heterogeneous integrated microrobot system. Transaction of The Japan Institute of Electronics Packaging 2019, 12, E18-009-1/7.

(C) 2020 by the authors; licensee MDPI, Basel, Switzerland. This article is an open access article distribute $\mathrm{d}$ under the terms and conditions of the Creative Commons by Attribution (CC-BY) license (http://creativecommons.org/licenses/by/4.0/). 